\title{
Optimization of the experimental conditions for structural studies of the second transmembrane domain from human wild-type \& mutant melanocortin-4 receptor
}

\author{
Ga-Ae Gang, Sung-Sub Choi, Tae-Joon Park, and Yongae Kim* \\ Department of Chemistry, Protein Research Center for Bio-Industry, \\ Hankuk University of Foreign Studies, Yongin 449-791, Korea \\ (Received Oct 30, 2010; accepted Dec 9, 2010)
}

\begin{abstract}
Human melanocortin-4 receptor (hMC4R) has a critical role in part of energy homeostasis, and their heterozygous mutations related in genetic cause of severe human obesity. In order to study the structure and function of these membrane proteins, it is important to prepare the samples. However, the preparation of transmembrane peptide is seriously difficult and time-consuming. Overexpression and purification of membrane proteins was reported to be difficult due to their innate insoluble and toxic properties. Among the many difficulties, the most important is the difficulty in obtaining sufficient quantities of purified protein. Recently, we succeed to produce large amounts of the second transmembrane domain from the wild-type hMC4R (wt-TM2) and D90N mutant hMC4R (m-TM2) and proposed the structural difference of them in membrane-like environments. In this paper, we demonstrate the optimization procedures to express and purify wt-TM2 or m-TM2 peptides, and solution NMR studies in different detergents to get high-resolution spectra were also described.
\end{abstract}

Keywords : melanocortin-4 receptor, solid-state NMR spectroscopy

\section{INTRODUCTION}

Melanocortin receptors are members of the rhodopsin family of 7-transmembrane, G-protein coupled receptors (GPCR). Each melanocortin receptors has differing specificities for melanocortins ${ }^{1,}$

* To whom correspondence should be addressed. E-mail : yakim@hufs.ac.kr 
${ }^{2}$. Among them, the melanocortin-4 receptor (MC4R) subtype has been highlighted recently by genetic studies in obese humans and mice, and is regarded as a potential drug target because of the importance of central melanocortinergic pathways in the regulation of feeding behavior and body weight $^{3}$. Several recent studies reported heterozygous mutations in MC4R gene were detected in $1 \sim 6 \%$ of the studied population of severe early-onset obese patients ${ }^{4-8}$. Especially, in a patient with severe early-onset obesity, it was detected a novel heterozygous mutation in the hMC4R gene resulting in an exchange of D90 to $\mathrm{N}$ located in the second transmembrane domain (TM2) that is highly conserved throughout the melanocortin receptor family9 .

Despite the growing interest in the role of MC4R in the control of mammalian energy homeostasis, the signal transduction mechanisms remain incompletely understood. One of the main reasons is that MC4R is a transmembrane protein, which spans from the internal to the external surface of the biological membrane. Generally, the preparation of transmembrane protein is seriously difficult, costly, and time-consuming due to a lot of experimental limitations ${ }^{10-12}$. The problem is the relatively low abundance of transmembrane proteins as compared with other cellular proteins, and the purification of them remains quite difficult because of the hindrance by inherent toxicity and aggregative nature of themselves. Even in the most successful cases, the over-expression and purification protocols are often distinct for each target.

To better understand the structure-activity relationship of the second transmembrane domain from the wild-type hMC4R (wt-TM2) and D90N mutant hMC4R (m-TM2), we succeed to produce 
large amounts of them through many purification tests for a long time. Especially, we expended a lot of times and effort on optimizing purification schemes. Recently, we reported the results of expression and purification, and proposed the structural difference of them in membrane-like environments ${ }^{13}$. In this paper, we demonstrate the optimization procedures to express and purify wtTM2 or m-TM2 peptides, and solution NMR studies in different detergents to get high-resolution spectra were also described.

\section{EXPERIMENTAL}

\section{Expression and purification of fusion protein}

hMC4R wt-/m-TM2 peptide were respectively constructed into pET-31b vector (Novagen Inc.) and expressed in E.coli C43(DE3) and C41(DE3) cells with isotope-enriched M9 minimal media. ketosteroid isomerase (KSI)-fused protein was induced by addition of 1mM IPTG at OD600 0.5 0.6, and cells were grown for additional $12 \mathrm{hrs}$ at $37{ }^{\circ} \mathrm{C}$. Harvested cells were lysed by ultrasonication. Cell lysates were centrifuged at 13,200 rpm for $30 \mathrm{~min}$ at $4{ }^{\circ} \mathrm{C}$. The pellets were dissolved under denaturing conditions containing $6 \mathrm{M}$ guanidine $\mathrm{HCl}$ for $5 \mathrm{hrs}$ at room temperature, and then loaded on Ni-NTA column. After washing column with 4 volumes of washing buffer, KSI-fused protein was

eluted with four volumes of elution buffer. To remove guanidine and salts, elutes were dialyzed 
against deionized water. The insoluble precipitates of KSI-fused proteins were collected and lyophilized.

\section{CNBr Cleavage}

Lyophilized fused protein was dissolved in 70\% formic acid, and cleavage to remove the fusion partner KSI and $\mathrm{His}_{6}$-tag form fusion protein was initiated by adding cyanogen bromide. Reaction mixture was incubated at room temperature under dark environments for 5 hrs. Reaction mixture after chemical cleavage was diluted five-fold with deionized water, and then dialyzed in a 1,000 MWCO membrane to remove the impurities, such as cyanogen bromide and formic acid. Finally, dialyzed solution was lyophilized. The purity of the isolated protein at each stages was confirmed by $12 \%$ tristricine polyacrylamide gel electrophoresis (PAGE).

\section{HPLC}

Final purification of wt-TM2 peptide was tested at different conditions using preparative reversed-phase HPLC system (Dionex, USA). Protein mixtures containing wt-TM2 were dissolved in four different solvents at a concentration of $3 \mathrm{mg} / \mathrm{ml}$; (1) $0.1 \%$ TFA (trifluoroacetic acid) in water, (2) TFE (trifluoroethanol), (3) 1:4 TFE:MC (methylene chloride), (4) 1:1 TFE / 0.1 \% TFA in water. The solutions were filtered using PTFE syringe filter, and then injected to HPLC equipped with a 
92 Optimization of the Experimental Conditions for structural studies of human melanocortin-4 receptor TM2

Delta Pak C4 column (7.8 × 300 mm, Waters). Peptides were eluted with a linear gradient of eluent A (95 \% water, $5 \%$ acetonitrile, and 0.1\% TFA) and B (5 \% water, $95 \%$ acetonitrile, and 0.1\% TFA). HPLC elution was monitored at $220 \mathrm{~nm}$ and $280 \mathrm{~nm}$ using a PDA detector at a flow rate of $3 \mathrm{ml} / \mathrm{min}$. Each fraction was pooled and identified by $12 \%$ tris-tricine PAGE.

\section{Double-dialysis}

Final purification of wt-TM2 peptide was conducted by using two different sizes of dialysis membrane simultaneously. Reaction mixture after cleavage was diluted five-fold with deionized water, and then transferred into a 10,000 MWCO dialysis membrane (diameter 22mm, flat width 34mm). This membrane containing reaction mixture was inserted into a 1,000 MWCO dialysis membrane (diameter 29mm, flat width $45 \mathrm{~mm}$ ). Deionized water or 14 \% FA (formic acid) was filled in the gap between two different sizes of dialysis membrane, and these were dialyzed against deionized water. After dialysis, each component inside and outside of 10,000 MWCO dialysis membrane was collected, lyophilized, and identified by $12 \%$ tris-tricine PAGE.

\section{Gel filtration chromatography}

For better purification of the hMC4R wt-/m-TM2 peptides, reaction mixture after cleavage was dissolved in buffer A (100 mM Na $\mathrm{HPO}_{4}, 4$ mM SDS, 1 mM EDTA, 1 mM NaN 3 , 20 mM DTT, pH 
7.4) and filtered through a $0.45 \mu \mathrm{m}$ pore size nylon syringe filter. The solution was separated by using a Sephacryl S200 column $(600 \times 26 \mathrm{~mm}$ with a bed volume of $320 \mathrm{ml})$ on an AKTA Prime system (GE Healthcare Biosciences, USA). The column was equilibrated and eluted with buffer A at a flow rate of $1 \mathrm{ml} / \mathrm{min}$. Fractions corresponding to the micellized hMC4R wt-/m-TM2 peptides were collected and dialyzed against deionized water at room temperature for five days using a 1,000 MWCO membrane tubing to remove the salts and SDS, followed by lyophilization. The purity of hMC4R wt-/m-TM2 peptides was identified by $12 \%$ aspartate PAGE.

\section{Solution NMR spectroscopy}

Micelle Samples were prepared by dissolving $1 \mathrm{mg}$ of ${ }^{15} \mathrm{~N}$-labeled hMC4R wt-/m-TM2 peptides in $300 \mu \mathrm{L}$ of $150 \mathrm{mM}$ perdeuterated SDS- $\mathrm{d}_{25}$ or $500 \mathrm{mM}$ perdeuterated DPC- $\mathrm{d}_{38}$ containing optimized NMR buffer (10 mM Na $\mathrm{HPO}_{4}, 1 \mathrm{mM} \mathrm{NaN}$, $40 \mathrm{mM}$ DTT, $90 \% \mathrm{H}_{2} \mathrm{O}$ and $\left.10 \% \mathrm{D}_{2} \mathrm{O}\right)$. Solution NMR experiments were performed on a Bruker AVANCE 800 spectrometer equipped with a tripleresonance 5-mm probe with three-axis field gradients. NMR measurement conditions were optimized by changing the $\mathrm{pH}$ value from 4.0 to 2.3 in DPC and SDS micelle system. The two-dimensional ${ }^{1} \mathrm{H} /{ }^{15} \mathrm{~N}$ HSQC spectra were acquired in phase-sensitive mode using the echo-antiecho method in the indirect dimension. Water resonance was suppressed using a water flip-back pulse and decoupling of

${ }^{15} \mathrm{~N}$ was achieved by GARP4 modulation. Spectra were collected with 128-256 increments (8-16 
94 Optimization of the Experimental Conditions for structural studies of human melanocortin-4 receptor TM2

scans each) in $\mathrm{t} 1$ and 1024 data points in t2 time domains, and data processing was performed using TOPSPIN 2.1 software (Bruker Biospin).

\section{RESULTS AND DISCUSSION}

\section{Expression and purification of fusion protein}

High-level expression of KSI fused MC4R wt-/m-TM2 peptides was induced by addition of 1 mM IPTG at $\mathrm{OD}_{600} 0.5$ (Fig. 1, lane 2). Peptide purity and identities at each experimental stages were checked by $12 \%$ aspartate PAGE, as shown in Fig. 1. Since KSI-fused proteins were found in inclusion body (Fig. 1, lane 4) of cells grown at $37^{\circ} \mathrm{C}$ in $\mathrm{M9}$ medium enriched with stable isotopes, purification was performed from insoluble fractions. Insoluble fusion protein could be fully solubilized by unfolding with $6 \mathrm{M}$ guanidine hydrochloride. His-tagged fused protein, KSI-(wt-/mTM2)-His ${ }_{6}$, was efficiently purified by Ni-NTA affinity chromatography and then refolded by dialysis. We could get the high purity fused protein as shown in Fig. 1 (lane 5), and the yield was 200-220 mg for fused protein of hMC4R wt-TM2 and 230-250 mg for that of hMC4R m-TM2 per liter of M9 media. 
(a)

(b)

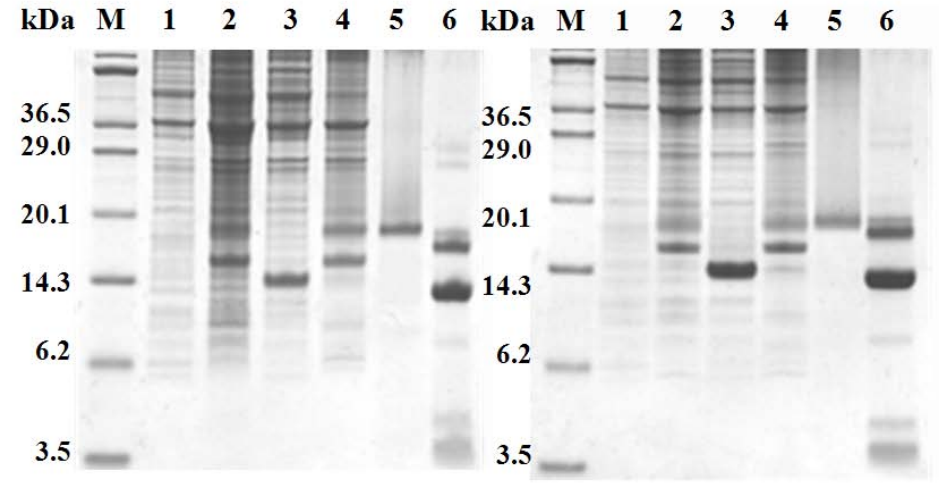

Figure. 1. $12 \%$ aspartate PAGE diagrams of the expression and purification for (a) hMC4R wt-TM2 and (b) hMC4R m-TM2. Lane M, marker; lane 1, total cell before induction; lane 2, total cell after induction; lane 3, soluble fraction after lysis; lane 4, insoluble inclusion bodies with a fusion protein after lysis; lane 5, eluates from column; lane 6, mixtures after cleavage.

\section{CNBr Cleavage}

KSI fused protein was chemically cleaved with $\mathrm{CNBr}$ to release the MC4R wt-/m-TM2 peptides (Fig. 1, lane 6). The band intensity of fused protein, $18 \mathrm{kDa}$ was significantly reduced and new bands corresponding to the fusion partner protein (13.5 kDa), hMC4R wt-/m-TM2 (3.2 kDa), and incompletely cleaved proteins (17 kDa and $4.2 \mathrm{kDa}$ ) appeared. The reaction mixture was diluted five- 
96 Optimization of the Experimental Conditions for structural studies of human melanocortin-4 receptor TM2

fold with deionized water, and then dialyzed overnight at room temperature against deionized water in 1,000 MWCO dialysis bags. Subsequently, the dialyzed mixture was lyophilized

\section{HPLC}

Final purification of wt-TM2 peptide was conducted at four different conditions using preparative reversed-phase HPLC system, as described above. Firstly, 15mg of dried protein mixtures after cleavage were dissolved in $15 \mathrm{ml}$ of $0.1 \%$ TFA in water, filtered, and injected into the column. Fig. 2(a) shows reversed-phase HPLC chromatogram in the first condition. The chromatogram shows two broad featureless peaks. From the result of Fig. 2(b), KSI fusion partner ( 14 kDa) and wt-TM2 peptide $(\sim 3.2 \mathrm{kDa})$ were eluted throughout the whole chromatography. Secondly, same amounts of protein mixtures were dissolved in $15 \mathrm{ml}$ of $100 \%$ TFE, filtered, and injected into the column. Fig. 2(c) shows reversed-phase HPLC chromatogram in the second condition. From the result of Fig. 2(d), it was not successful because of poor separation. Thirdly, same amounts of protein mixtures were dissolved in $15 \mathrm{ml}$ of 1:4 TFE:MC, filtered, and injected into the column. Some peaks were separated in the chromatogram of Fig. 2(e), but wt-TM2 peptide was still not purified based on the result of Fig. 2(f). Finally, we executed HPLC purification by dissolving the same amounts of protein mixtures in $15 \mathrm{ml}$ of 1:1 TFE / $0.1 \%$ TFA in water. Chromatogram and gel pattern under this condition (Fig. 2(g), (h)) was quite similar to the results of the second condition (Fig. 2(c), (d)). In fact, we made many 
other efforts to purify wt-/m-TM2 peptide by using reversed-phase HPLC under lots of conditions, such as changing the solvent, gradient condition, column etc. The most difficult problem to get over is its insoluble and aggregative property under the experiments.

(a)

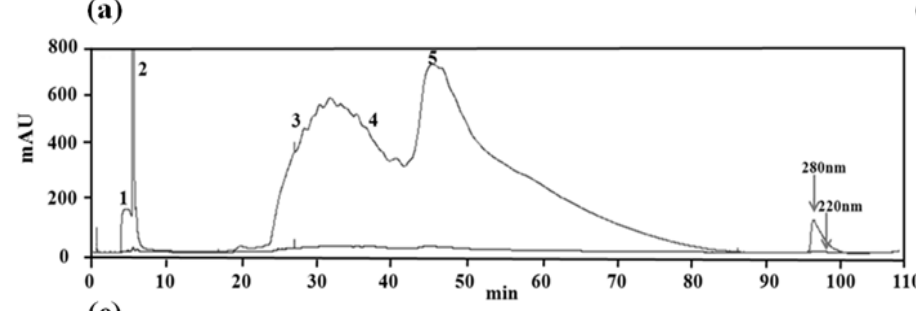

(c)

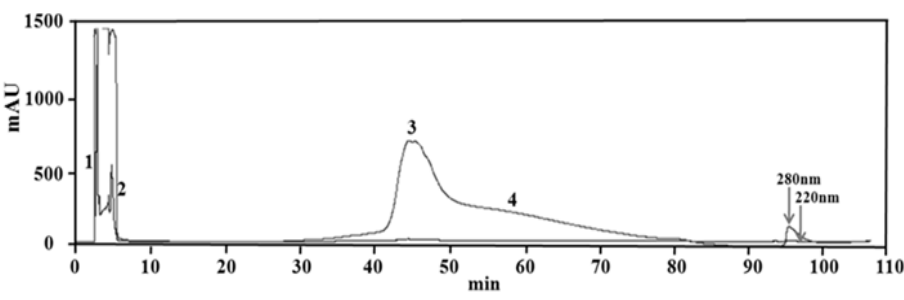

(e)
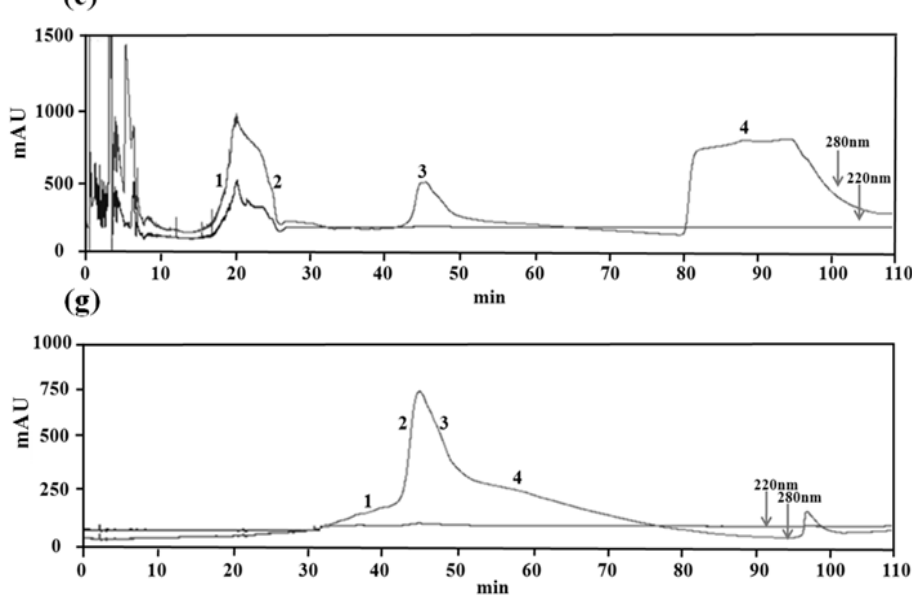

(b)

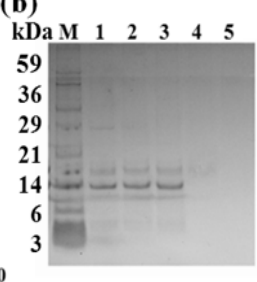

(d)

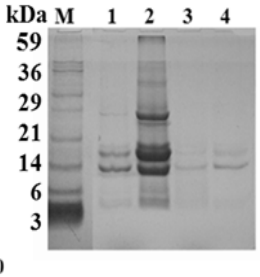

(f)

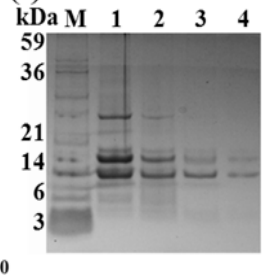

(h)

$\begin{array}{llllll}\text { kDa } & \text { M } & 1 & 2 & 3 & 4\end{array}$

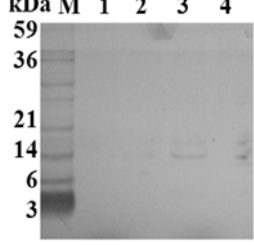

Figure. 2. HPLC chromatogram of wt-MC4R-TM2 sample dissolved in (a) $0.1 \%$ TFA in water (c) $100 \%$ TFE (e) 1:4 TFE:MC (g) 1:1 TFE / $0.1 \%$ TFA in water at a concentration of $3 \mathrm{mg} / \mathrm{ml}$. 
98 Optimization of the Experimental Conditions for structural studies of human melanocortin-4 receptor TM2

Peptides were eluted with a linear gradient of ACN in water and $0.1 \%$ TFA on the C4 column. Eluent A: $5 \%$ dd $_{2} \mathrm{O}, 95 \%$ ACN, $0.1 \%$ TFA, Eluent B: $5 \% \mathrm{ddH}_{2} \mathrm{O}, 95 \%$ ACN, $0.1 \%$ TFA. (b), (d), (f), (h) shows $12 \%$ tris-tricine PAGE diagrams from HPLC fractions of (a), (c), (e), and (g), respectively.

\section{Double-dialysis}

Final purification of wt-TM2 peptide was performed by using two different sizes of dialysis membrane simultaneously, as described above. Fig. 3 shows the result of $12 \%$ tris-tricine PAGE to identify each composition inside or outside of 10,000 MWCO dialysis membrane after doubledialysis. Lane 1 and 2 show protein profiles from fraction inside and outside of 10,000 MWCO dialysis membrane when deionized water was filled in the gap between two different sizes of dialysis membrane. And lane 3 and 4 show protein profiles from composition inside and outside of 10,000 MWCO dialysis membrane when $14 \%$ FA was filled in the gap between two different sizes of dialysis membrane. In both cases, fractions inside of 10,000 MWCO membrane (Fig. 3 lane 1, 3) contained all component from the cleavage reaction mixture, but the band corresponding to KSI at 14 $\mathrm{kDa}$ in lane 4 was less intense than that in lane 2. That is because KSI was slipped out of inner dialysis membrane in the case of water filled system in a gap between two different size of MWCO membrane bag. We found the presence of KSI with wt-TM2 peptide ( 3.2 kDa) from a fraction 
outside of inner membrane only in the case of water filled system (Fig. 3 lane 2). In the case of $14 \%$ FA filled system in a gap between two different size of MWCO membrane bag, fraction outside of inner membrane (Fig. 3 lane 4) contained relatively large amount of wt-TM2 peptide with some impurities. If we further improve this purification method, it will be a good technique to separate different size proteins or peptides.

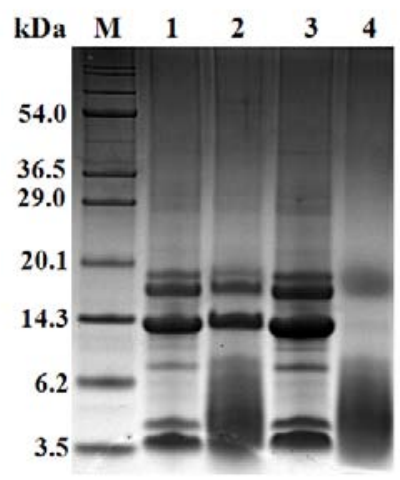

Figure. 3. $12 \%$ tris-tricine PAGE to identify each fraction inside or outside of 10,000 MWCO dialysis membrane after double-dialysis. Lane M, marker; Lane 1 and 2 show protein inside and outside of 10,000 MWCO dialysis membrane when deionized water was filled in the gap between two different sizes of dialysis membrane. Lane 3 and 4 show protein inside and outside of 10,000 MWCO dialysis membrane when $14 \%$ FA was filled in the gap between two different sizes of dialysis membrane.

\section{Gel filtration chromatography}


100 Optimization of the Experimental Conditions for structural studies of human melanocortin-4 receptor TM2

Human MC4R wt-/m-TM2 peptides were finally purified by gel filtration chromatography on a a Sephacryl S200 column (26/60) equilibrated with buffer A. A chromatogram obtained from the FPLC UV detector at $280 \mathrm{~nm}$, is shown in Fig. 4 (a), (c). Unfortunately, hMC4R wt-/m-TM2 peptides could not be detected in the FPLC chromatogram recorded at $280 \mathrm{~nm}$ because they are integral membrane proteins which are very hydrophobic. Therefore, we analyzed each fraction from the gel filtration by $12 \%$ aspartate PAGE. From the gel pattern, we found that the micellized hMC4R wt-/mTM2 peptides were eluted from 280 to 320 min. Every fraction were collected and dialyzed to remove the salts and SDS, followed by lyophilization. The final yield of the purified peptides was 5 $8 \mathrm{mg}$ per liter of M9 culture.

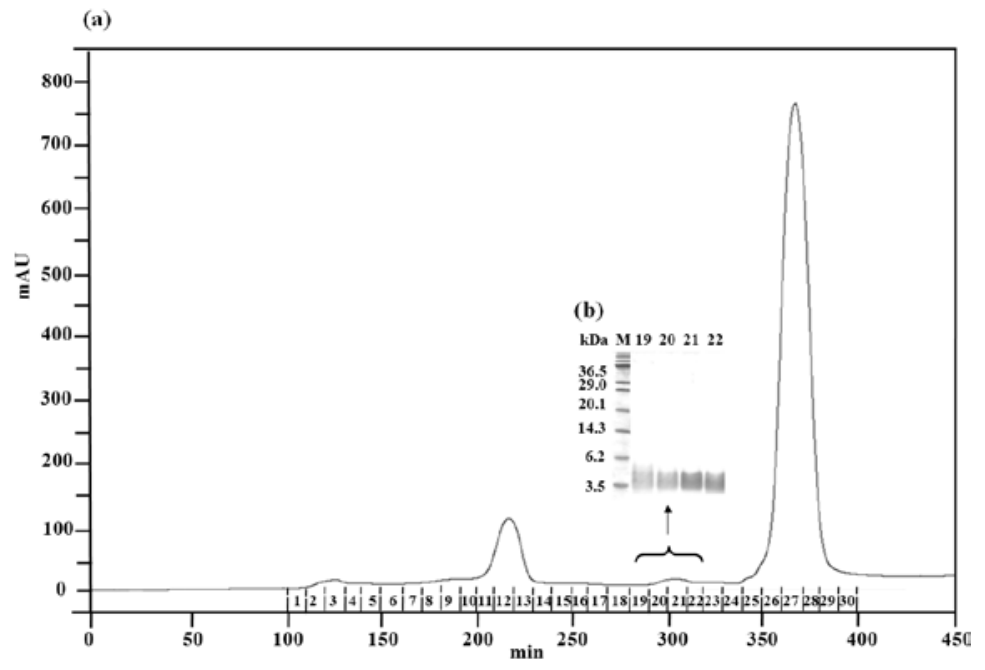




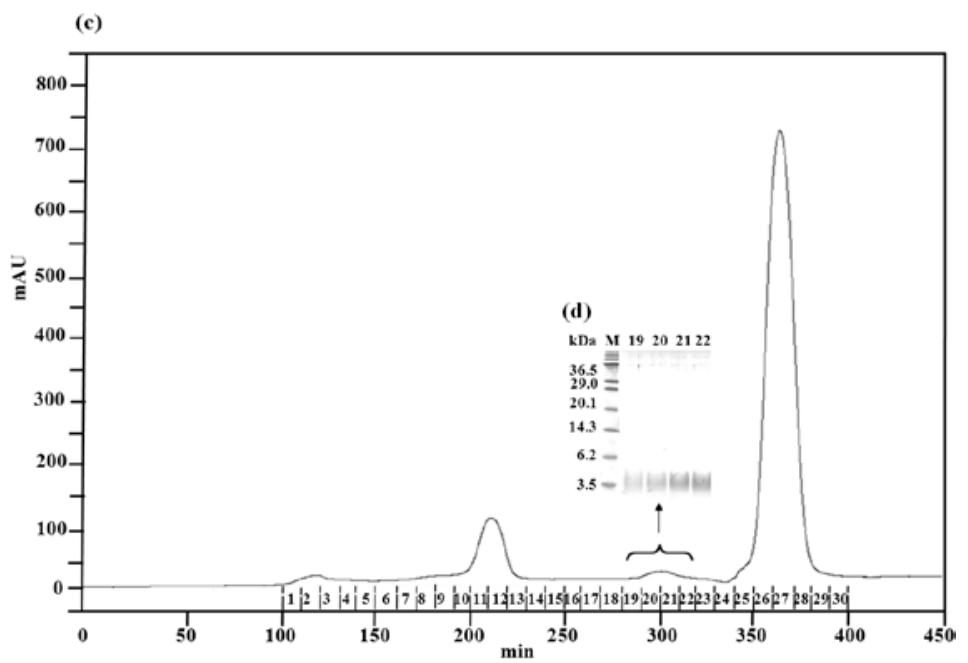

Figure. 4. FPLC chromatograms for purified (a) hMC4R wt-TM2 and (c) hMC4R m-TM2 peptides on a gel filtration column (Sephacryl S200). Fractions corresponding to the (a) hMC4R wt-TM2 peptide and (c) hMC4R m-TM2 peptides were analyzed by 12\% aspartate PAGE in (b) and (d), respectively.

\section{Solution NMR spectroscopy}

Purified wt-/m-TM2 peptides were dissolved in two different micelle systems, as described above. Even though under the micelle system, the peptides were not clearly dissolved at higher $\mathrm{pH}$ than $\mathrm{pH}$ 4.5. Under DPC micelle condition, abnormal broadening or clustering of resonances was detected, which is warning sign that the polypeptide is aggregated or improperly folded. So we tried to change the $\mathrm{pH}$ value from 4.0 to 2.3, as shown in Fig. 5. Nevertheless, we could not get the highresolution NMR spectra. Under SDS micelle condition, we could get the high resolution 2D HSQC 
spectra, as shown in Fig. 6. The majority of the ${ }^{1} \mathrm{H}-{ }^{15} \mathrm{~N}$ cross-peaks for each nitrogen bound hydrogen atom lie between 6.7 and $8.9 \mathrm{ppm}$, which is typical of ordered helical membrane proteins in micelles. Interestingly, the number of resonances in the ${ }^{1} \mathrm{H} /{ }^{15} \mathrm{~N}$ 2D HSQC spectrum of hMC4R wtTM2 peptide (Fig. 6 (a)) is much higher than that of hMC4R m-TM2 peptide (Fig. 6 (b)) although the peptide sequence differing by only one residues. That might be because of different oligomeric form between hMC4R wt-TM2 and m-TM2.

(a)

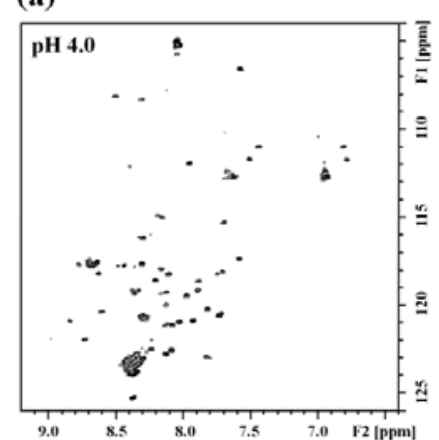

(c)

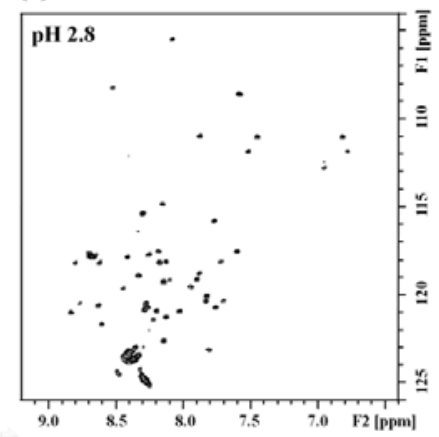

(b)

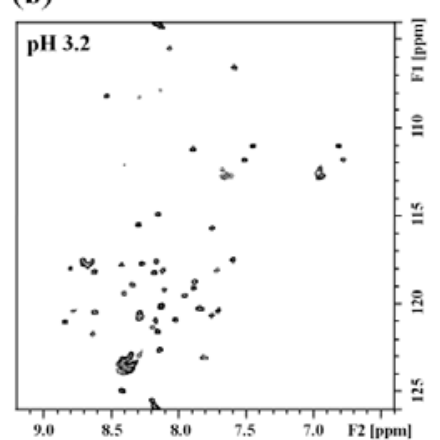

(d)

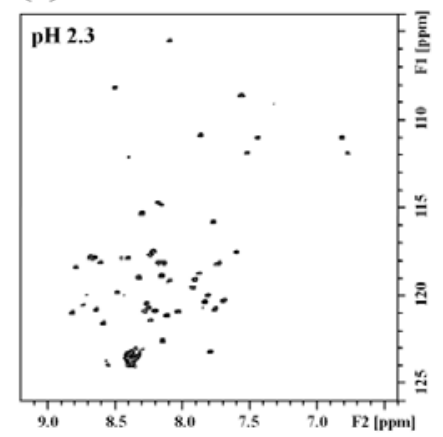

Figure. 5. ${ }^{1} \mathrm{H} /{ }^{15} \mathrm{~N}$ 2D HSQC spectra of hMC4R m-TM2 measured at (a) $\mathrm{pH} 4.0$, (b) $\mathrm{pH} 3.2$, (c) $\mathrm{pH}$ 2.8, and (d) pH 2.3 under DPC micelle condition. 

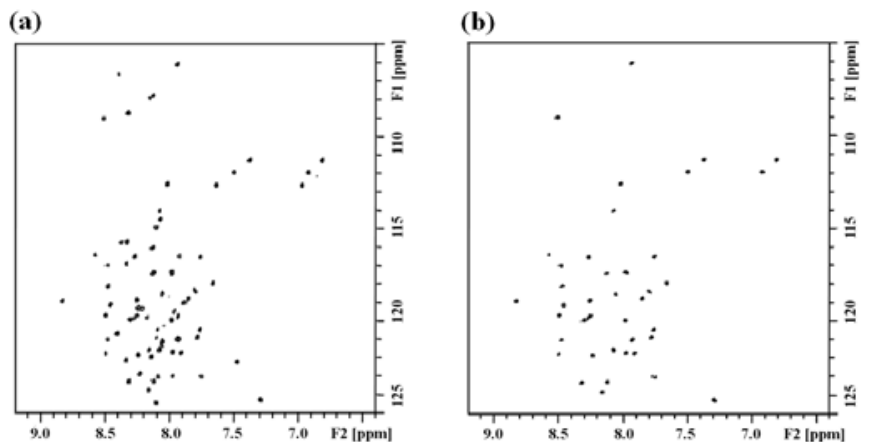

Figure. 6. ${ }^{1} \mathrm{H} /{ }^{15} \mathrm{~N}$ 2D HSQC spectra of (a) hMC4R wt-TM2 and (b) hMC4R m-TM2 under SDS micelle condition

\section{CONCLUSION}

In this paper, we demonstrate the optimization of expression, purification, and NMR measurement condition for structural studies of hMC4R wt-/m-TM2 peptides. The several methods used in this study will be helpful to produce other transmembrane peptides. Milligram quantities of hMC4R wt-/m-TM2 peptides were purified in micelle state by using gel filtration chromatography. As might have been expected, we could found the structural difference between purified hMC4R wtTM2 and D90N m-TM2 peptide in SDS micelle condition by using solution NMR spectroscopy.

\section{Acknowledgment}

This work was supported by Basic Science Research Program through the National Research Foundation of Korea(NRF) funded by the Ministry of Education, Science and Technology(2010- 
104 Optimization of the Experimental Conditions for structural studies of human melanocortin-4 receptor TM2

0021954) and the GRRC Program of Gyeonggi Province [GRRC HUFS 2010-B01]. This study made use of the NMR facility which is supported by NMR Research Program of the Korea Basic Science Institute (to YHJ).

\section{REFERENCES}

1. J. Voisey, L. Carroll, A. van Daal, Current Drug Targets 4, 586. (2003).

2. M. E. Hadley, and R. T. Dorr, Peptides 27, 921. (2006).

3. K. G. Mountjoy, M. T. Mortrud, M. J. Low, R. B. Simerly, and R. D. Cone, Mol Endocrinol. 8, 1298. (1994).

4. I. S. Farooqi, G. S. Yeo, J. M. Keogh, S. Aminian, S. A. Jebb, G. Butler, T. Cheetham, and S. O’Rahilly, J. Clin. Invest. 106, 271. (2000).

5. C. Vaisse, K. Clement, E. Durand, S. Hercberg, B. Guy-Grand, and P. Froguel, J. Clin. Invest. 106, 253. (2000).

6. I. S. Farooqi, J. M. Keogh, G. S. Yeo, E. J. Lank, T. Cheetham, and S. O’Rahilly, N. Engl. J. Med. 348, 1085. (2003).

7. C. Lubrano-Berthelier, E. Durand, B. Dubern, A. Shapiro, P. Dazin, J. Weill, C. Ferron, P. Froguel, and C. Vaisse, Hum. Mol. Genet. 12, 145. (2003).

8. B. Dubern, K. Clement, V. Pelloux, P. Froguel, J. Girardet, B. Guy-Grand, and P. Tounian, J. Pediatr. 139, 204. (2001).

9. B. Heike, K. Heiko, E. Andrea, C. Vladimir, G. Thomas, and G. Annette, Diabetes 52, 2984. (2003).

10. R. Grisshammer, and C. G. Tate, Q. Rev. Biophys. 28, 315. (1995).

11. P. J. Loll, J. Struct. Biol. 142, 144. (2003).

12. C. G. Tate, and R, Grisshammer, Trends Biotechnol. 14, 426. (1996).

13. T. J. Park, S. S. Choi, G. A. Gang, and Y. Kim, Protein Expr. Purif. 62, 139. (2008). 